\title{
Ocular Pathology and Occasionally Detectable Intraocular Severe Acute Respiratory Syndrome Coronavirus-2 RNA in Five Fatal Coronavirus Disease-19 Cases
}

\author{
Aja Reinhold ${ }^{a} \quad$ Alexandar Tzankov ${ }^{b}$ Matthias S. Matter ${ }^{b}$ Daniela Mihic-Probst ${ }^{c}$ \\ Hendrik P.N. Scholl ${ }^{a, d}$ Peter Meyer ${ }^{a, b}$ \\ aDepartment of Ophthalmology, University of Basel, Basel, Switzerland; 'blnstitute of Medical Genetics and \\ Pathology, University of Basel, Basel, Switzerland; 'Institute of Pathology and Molecular Pathology, University of \\ Zurich, Zurich, Switzerland; 'Institute of Molecular and Clinical Ophthalmology Basel, Basel, Switzerland
}

\section{Keywords \\ Ocular pathology · Syndrome coronavirus-2 - Coronavirus disease 2019 · Vasculopathy · Angiotensin-converting enzyme 2}

\begin{abstract}
Introduction: In December 2019, the severe acute respiratory syndrome coronavirus-2 (SARS-CoV-2) pandemic broke out. The virus rapidly spread globally, resulting in a major world public-health crisis. The major disease manifestation occurs in the respiratory tract. However, further studies documented other systemic involvement. This study investigates histopathologic eye changes in postmortem material of coronavirus disease 2019 (COVID-19) patients. Methods: Sections of formalin-fixed, paraffin-embedded eyes from 5 patients (10 eyes) who died of COVID-19 at the University Hospital in Basel were included. Gross examination and histological evaluation were performed by 3 independent ophthalmopathologists. Immunohistochemical staining was performed using antibodies against fibrin, cleaved caspase 3 , and ACE-2. Five enucleated eyes of patients not infected with SARS-CoV-2 served as control group. All cases have been studied for presence of SARS-CoV-2 RNA by means of reverse transcription PCR and RNA in situ hybridization (ISH).
\end{abstract}

The choroidal vessels of one case were analyzed with electron microscope. Results: Ophthalmopathologically, 8 eyes from 4 patients displayed swollen endothelial cells in congested choroidal vessels. No further evidence of specific eye involvement of SARS-CoV-2 was found in any of the patients. In the 8 eyes with evidence of changes due to SARS-CoV-2, immunohistochemical staining demonstrated fibrin microthrombi, apoptotic changes of endothelial and inflammatory cells. In control eyes, ACE-2 was detectable in the conjunctiva, cornea, retina, and choroidea and displayed significantly lower amounts of stained cells as in COVID-19 eyes. SARS-CoV-2 RNA was detectable in both bulbi of $2 / 5$ patients, yet ISH failed to visualize viruses. Electron microscopy showed no significant results due to the artifacts. Discussion/Conclusion: As already described in other organs of COVID-19 patients, the ophthalmological examination revealed-microthrombi, that is, hypercoagulation and vasculopathy most probably due to endothelial damage. A possible viral spread to the endothelial cells via ACE-2 provides one pathophysiological explanation. The expression of ACE2 receptors in the conjunctiva hints toward its susceptibility to infection. To what extend eyes, function is disrupted by SARS-CoV-2 is subject to further studies, especially in the clinic.

(c) 2021 The Author(s)

Published by S. Karger AG, Basel
C 2021 The Author(s).

Published by S. Karger AG, Basel

This article is licensed under the Creative Commons Attribution 4.0 International License (CC BY) (http://www.karger.com/Services/ OpenAccessLicense). Usage, derivative works and distribution are permitted provided that proper credit is given to the author and the original publisher.
Correspondence to:

Aja Reinhold, aja.reinhold@usb.ch

Peter Meyer, peter.meyer@usb.ch 


\section{Introduction}

In December 2019, the severe acute respiratory syndrome coronavirus-2 (SARS-CoV-2) pandemic broke out and resulted in a public-health crisis with over 15.2 million infections and over 620,000 deaths in July 2020. Certain comorbidities such as hypertension, diabetes, and cardiovascular diseases are associated with increased mortality [1]. It is already known that the SARS-CoV-2 enters cells via the angiotensin-converting enzyme 2 (ACE-2) [1-3]. ACE-2 is found not only in epithelia of the lung, kidney, heart, and intestines but also in vascular endothelial cells in almost all organs [4]. Compounds of the renin-angiotensin system and especially ACE have been detected in the eye (cornea, ciliary body, retina, and choroidea) [5]. Eye involvement in coronavirus disease 2019 (COVID-19) patients is, therefore, theoretically possible but has not yet been described in the literature.

Ocular manifestations of SARS-CoV-2 have been reported in animals. Conjunctivitis, anterior uveitis, and severe complications such as retinitis or optic neuritis have been described in felines and mice [6]. SARS-CoV-2 conjunctivitis has already been described in human [7].

The aim of our study was the description of histological eye changes in postmortem material of patients, who suffered from COVID-19 and the detection of viruses by reverse transcription PCR (RT-PCR), in situ hybridization (ISH), and electron microscopy. ACE-2, fibrin microthrombi, and caspase 3 expressions were specifically investigated using immunohistochemistry, and correlations with systemic organ damage were performed.

\section{Materials and Methods}

Case Selection, Consent, and Ethical Approval

In 5 patients suffering from COVID-19 in Basel (Switzerland), full autopsies including eye globe removal were performed and evaluated by 3 independent ophthalmopathologists. All examined patients died of respiratory failure. The mean postmortem delay was $20 \mathrm{~h}(11-26 \mathrm{~h})$.

Five enucleated eyes of non-COVID-19 autopsies served as control group. These 3 patients died of cholangiosepsis, adenocarcinoma of the lung, and bronchopneumonia. Consents of the next of kin were obtained that the organs collected at autopsy may be used for research purposes.

\section{Ancillary Techniques}

Eye Fixation, Embedding, Sectioning, and Staining

Eye globes were fixed in $4 \%$ buffered formaldehyde solution for $48 \mathrm{~h}$ at room temperature. Prior to paraffin embedding, the superior calottes (horizontal meridian) were removed. The remaining pupil-optic section included the cornea, anterior chamber and its angles, lens iris, ciliary body, and a representative portion of the choroid and retina (including macular region). The optic disc and optic nerve were embedded, too. 4 - $\mu$ m-thick consecutive sections were sliced from each eye, stained with hematoxylin and eosin as well as applying periodic acid-Schiff reagent, elastic van Gieson, and Masson's trichrome techniques.

Immunohistochemical staining was performed using polyclonal antibodies for fibrin (1:100'000 diluted polyclonal rabbitanti-human antibody A0080 from Dako, Glostrup, Denmark), cleaved (active) caspase 3 (polyclonal rabbit-anti-human ready-touse antibody PP 229 AA from Biocare, Pacheco, CA, USA), and ACE-2 (1:10'000 diluted polyclonal rabbit-anti-human antibody, Abcam ab15348, Cambridge, UK). Immunohistochemistry was performed on the Benchmark Ultra automated immunostainer from Roche-Ventana according to the current accredited staining protocols of our institute. ISH was performed applying the 845701 RNAscope probe-V-nCoV2019-S-sense and duplexed with the 859151 RNAscope probe-V-nCoV2019-orf1ab-sense and with the RNAscope 2.5. LS detection kit (brown) from Advanced Cell Diagnostics (Hayward, CA, USA) on the Leica Biosystems BOND III autostainer (Muttenz, Switzerland) as per manufacturer's protocol.

A quantitative RT-PCR assay was performed on all samples to detect the presence of SARS-CoV-2 RNA as previously described [8]. Viral copy number was calculated according to standard methods and normalized to number of RNAse-P molecules. The positive result of patients 4 and 5 had been confirmed by independent cycles of each of both eyes.

For detection of SARS-CoV-2, RNA was first isolated from formalin-fixed and paraffin-embedded tissue by using the Maxwell RSC RNA FFPE Kit (Promega, Madison, WI, USA) according to manufacturer. Afterward, TaqMan RT-PCR was performed by using the TaqMan 2019-nCoV Control Kit v1 (ThermoFisher Scientific, Catalog Number A47533) to target 3 different viral genomic regions (ORF1ab, $\mathrm{S}$, and $\mathrm{N}$ protein) and the human RPPH1 gene (RNAse-P). According to the manufacturer's protocol, a CT value below 37 in at least 2 out of 3 viral genomic regions was considered positive. A case was considered negative if CT values were above 40. Values between 37 and 40 were considered undetermined and the assay was repeated. Samples were always run in duplicates. Samples showing positivity were repeated independently for confirmation. Electron microscopy was performed on choroid tissue of case 1 (left eye) according to standard institute protocols utilizing a transmission electron microscope (Morgagni 268D, FEI Co., Hillsboro, OR, USA).

\section{Results}

\section{Clinical Records and Medical Condition}

Case 1

A 67-year-old female died 14 days after initial symptoms of COVID-19 with focal superposed pneumonia. She suffered from secondary progressive multiple sclerosis. Ophthalmologically, the patient had a bilateral optic atrophy with reduction of visual acuity. 
Table 1. Pathological findings on HE stained slides

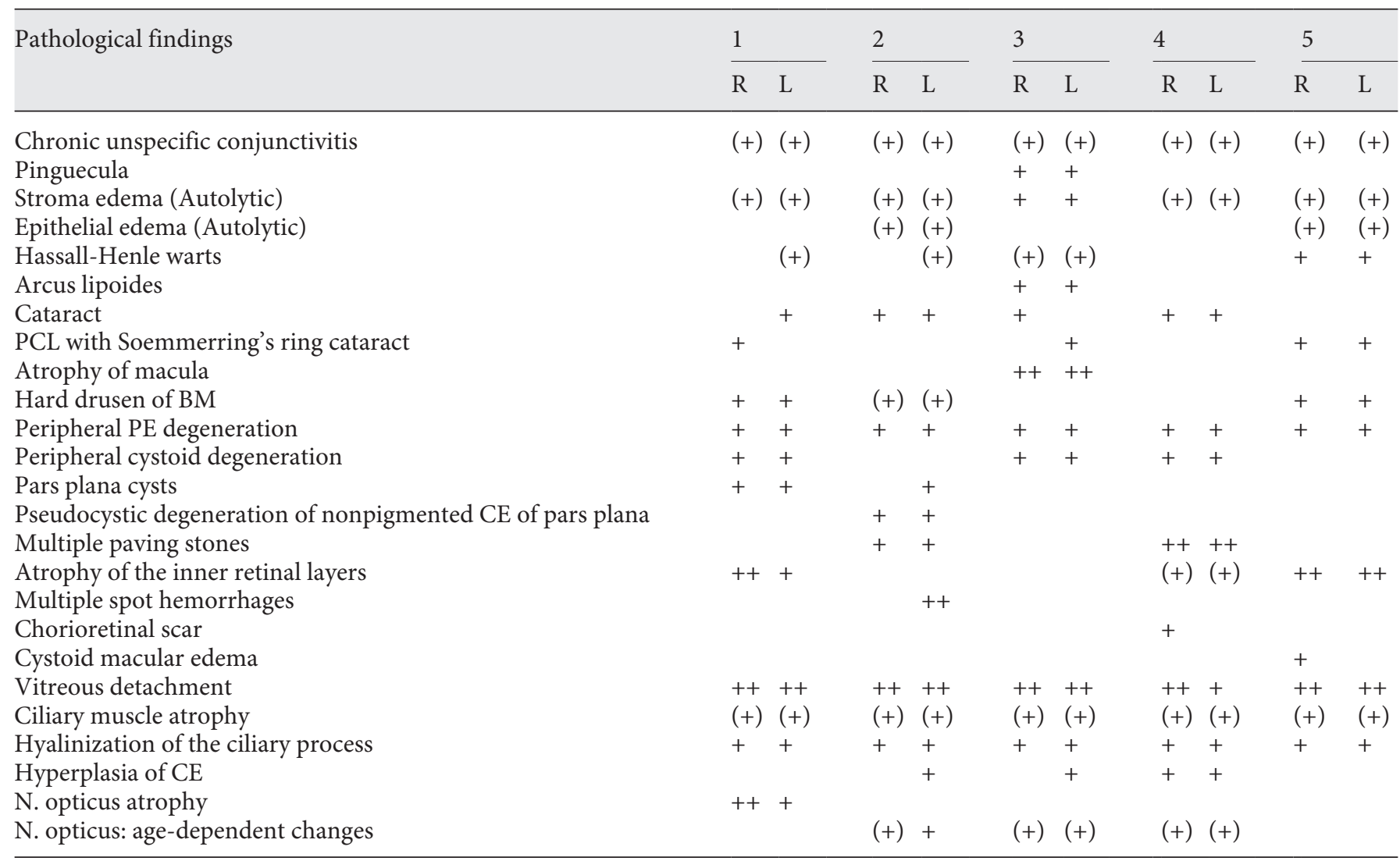

BM, Bruch's membrane; PE, pigmented epithelium; CE, ciliary epithelium; N, nerves; HE, hematoxylin and eosin.

Case 2

A 85-year-old male patient died 3 days after initial symptoms of COVID-19. He suffered from a therapy-associated myeloid neoplasia that fulfilled criteria of a myelodysplastic syndrome with blast excess type 1 and myelofibrosis. Additionally, he had diabetes mellitus type 2, cardiac decompensation, arterial hypertension, a severe obstructive sleep apnoea syndrome, and an incidental carcinoma of the prostate. The ophthalmologic findings indicated a visual impairment due to epiretinal fibrosis and beginning of cataract.

\section{Case 3}

A 95-year-old male patient died 4 days after the beginning of COVID-19 and had a severe superposed pneumonia. He suffered from Parkinson's syndrome with dementia and asympathicotonic orthostatic dysregulation as well as coronary, valvular, and hypertensive cardiopathy. Ophthalmologically, an age-related macular degeneration was known.

Ocular Pathology in SARS-CoV-2

\section{Case 4}

The fourth male patient died 23 days after the initial COVID-19 symptoms at the age of 66 years. He suffered ventilator-associated pneumonia. Clinically, he also had arterial hypertension and obstructive sleep apnoea syndrome. In addition, slight reduction of visual acuity with papillary anisoexcavation on the left side more than on the right side and a beginning of cataract were diagnosed.

Case 5

A 73-year-old male patient died of COVID-19 with superposed bilateral pneumonia 5 days after the onset of disease. He suffered from chronic obstructive pulmonary disease, chronic kidney failure, diabetes mellitus type 2, arterial hypertension, and genetic thrombophilia. In addition, a progression of osseous and lymphogenic metastasizing adenocarcinoma of the prostate was known. Ophthalmologically, he displayed a bilateral venous branch occlusion and a moderate nonproliferative diabetic retinopathy. 


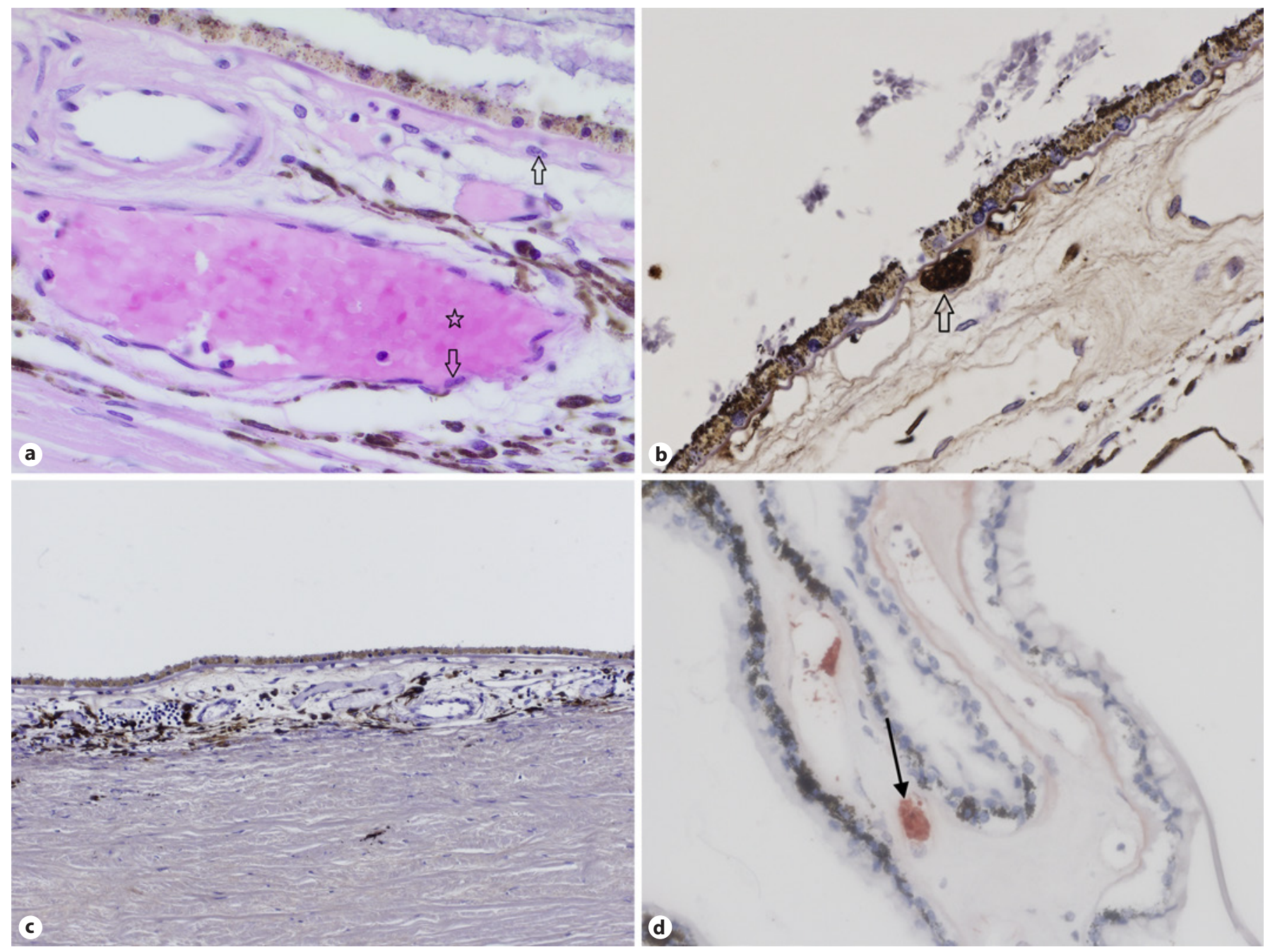

Fig. 1. Choroid vessels of case 1 (a, b), control group (c), and case 4 (d): microthombi in choriocapillaris and blood congestions in larger choroidal vessel (star), swollen endothelial cells of choriocapillaris (arrows) ( $\times 40$ objective; HE stain) (a); fibrin microthombus in choriocapillaris (arrow) ( $\times 60$ objective; fibrin immunhistochemistry) (b); control group ( $\times 20$ objective; fibrin immunhistochemistry) (c); and fibrin microthombus in ciliary body (arrow) ( $\times 40$ objective; fibrin immunhistochemistry) $(\mathbf{d})$. HE, hematoxylin and eosin.

\section{Ophthalmopathologic Findings}

Detailed histopathologic findings are listed in Table 1. All eyes showed only patient-specific changes corresponding to the respective pre-existing ocular conditions and age-dependent changes of the eyes. There was no evidence of SARS-CoV-2-associated inflammatory processes in neural tissues, in the iris, or in corneal tissues. The perilimbal conjunctival stroma showed a supremely mild inflammatory cell infiltration with a slight edematous change of the loose connective tissue stroma.

In both eyes of 4 patients (cases 1, 3, 4, and 5), we found distinct accumulation of inflammatory cells (mononuclear and neutrophilic) in the lumen and intima of congest- ed capillaries of the choriocapillaris. Some endothelial cells in this area were swollen distinctly (shown in Fig. 1a).

\section{Immunohistochemistry}

Fibrin

Four of 5 patients (cases 1, 3, 4, and 5) displayed multiple small fibrin thrombi in the choriocapillaris and less in the larger choroidal vessels, the retinal vessels, and some in the ciliary vessels (shown in Fig. 1b, d; Table 2). Sporadically, fibrin thrombi were found in the episclera and the intraneural septum vessels of the optic nerve. In one case (case 1, right eye) a microthrombus could be described in the circle of Zinn and Haller. The small vessels 
Table 2. Presence of microthrombi on fibrin stained slides

\begin{tabular}{llllll}
\hline Case & Site & Ciliary vessels & Retinal vessels & Choriocapillaris & Choroidal vessels \\
\hline 1 & R & - & - & +++ & + \\
& L & + & + & +++ & ++ \\
2 & R & - & - & - & - \\
3 & L & - & - & - & + \\
& R & + & + & +++ & + \\
4 & L & + & + & ++ & - \\
& R & + & + & ++ & + \\
5 & L & + & + & + & + \\
& R & + & + & + & Choroidal vessels \\
\hline Controls & L & - & + & Choriocapillaris & - \\
\hline 1 & Site & Ciliary vessels & Retinal vessels & - & - \\
2 & R & - & - & - & - \\
3 & L & - & - & - & - \\
4 & R & - & - & - & - \\
5 & L & - & - & - & - \\
\hline
\end{tabular}

,$+ 1-5$ vessels per slide section;,$++ 5-10$ vessels per slide section;,$+++>10$ vessels per slide section.

Table 3. RT-qPCR assay results in comparison to lung

\begin{tabular}{lll}
\hline Patient & $\begin{array}{l}\text { Eye } \\
\text { CT-values for ORF1ab, S, and N protein }\end{array}$ & $\begin{array}{l}\text { Lung } \\
\text { Highest CT-values for ORF1ab, S, and N protein }\end{array}$ \\
\hline 1 & Right and Left: negative & negative \\
2 & Right and Left: negative & positive $(19 ; 19 ; 19)$ \\
3 & Right and Left: negative & positive $(17 ; 17 ; 17)$ \\
4 & Right: positive $(34 ; 32 ; 32)$ & \\
& Left: positive $(35 ; 33,33)$ & positive $(17 ; 17 ; 17)$ \\
5 & Right: positive $(33 ; 34 ; 35)$ & \\
\hline
\end{tabular}

RT-qPCR, quantitative reverse transcription PCR.

of the conjunctiva, iris, and central retina vessels contained no fibrin thrombi.

No eye globes of the control group showed any fibrin thrombi (shown in Fig. 1c). Due to postmortem change, in 2 patients increased cruor was found in the choroidal vessels.

\section{Cleaved (Active) Caspase 3}

In both eyes of 4 patients (cases 1, 3, 4, and 5), cleaved caspase 3 expression (which functions as an apoptosis executor) was mainly found in endothelial cells and inflammatory cells infiltrating the intima of the choriocapillaris (shown in Fig. 2a). Increased apoptotic activity in neural tissue of the retina and the optic nerve was noted. In the control group, only single stained cells were detectable in choroidal vessels (shown in Fig. 2b)

\section{ACE-2}

The expression of ACE-2 was performed by semiquantitative immunohistochemistry. A spot-like staining of the thin endothelial cells of the choriocapillaris and some of the larger vessels were observed (shown in Fig. 2c). In addition, the photoreceptor and the endothelial cells of small retinal vessels within the nerve fiber layer can be marked more in a diffuse manner. In the ciliary body, nonpigmented epithelial cells presented intracel- 


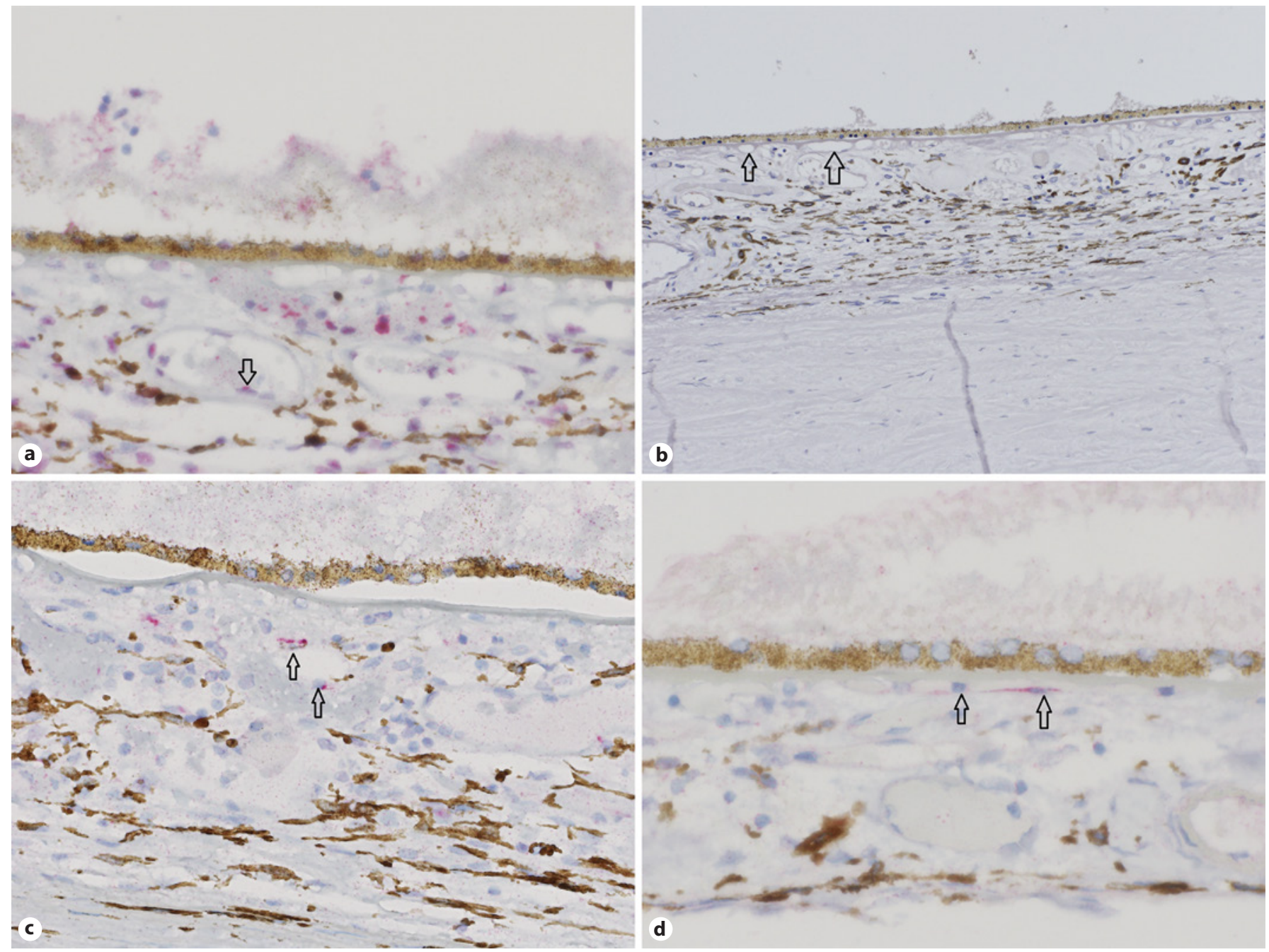

Fig. 2. Immunohistochemical staining. a Immunohistochemical staining of cleaved caspase 3 in endothelial cell of a choroid vessel (arrow) ( $\times 40$ objective; caspase 3 ). b Immunohistochemical staining of cleaved caspase 3 (control group) no staining is shown in choriocapillaris (arrows) or lager vessels of choroid ( $\times 20$ objective; caspase $3)$. c Immunohistochemical staining of ACE- 2 in endothelial cells of choriocapillaris (arrows) $(\times 40$ objective; ACE-2). d Immunohistochemical staining of ACE-2 (control group) in endothelial cells of choriocapillaris (arrows) ( $\times 60$ objective; ACE- 2 ).

lular staining for ACE-2 as well as the corneal epithelium, endothelium, and the conjunctival epithelium (shown in Fig. 3). Central vessel within the optic nerve and the ciliary vessels were negative. In 3 patients, the endothelia of small retinal vessels were slightly more positive for ACE2 compared to the control eyes. It seems that the expression of ACE-2 in choriocapillaris is higher than in the control group (shown in Fig. 2d).

PCR and in situ Hybridization

Postmortem RT-PCR analysis of the bulbi yielded positive results, defined as $\mathrm{C}_{\mathrm{T}}$-values below 37 , in both eyes of 2 patients (shown in Table 3 ). In case 4, RT-PCR was positive for all 3 viral regions (ORF1ab, $\mathrm{S}$, and $\mathrm{N}$ protein), and $\mathrm{C}_{\mathrm{T}^{-}}$ values were between 32 and 35 . In case 5, RT-PCR was also positive for all 3 viral regions tested, and $\mathrm{C}_{\mathrm{T}}$-values ranged between 33 and 36 . However, amount of viral RNA in the eyes was considerably lower than in the lungs of the same patient, where $\mathrm{C}_{\mathrm{T}}$-values were as low as 15 for case 4 and as low as 17 for case 5 . A rough estimate of viral copies after comparison with standard control indicated that viral copies in the eyes are $10^{1}-10^{3}$ times lower than in the lungs. Viral RNA remained undetectable in any case by ISH, which further supported the low viral amount in the eyes. 


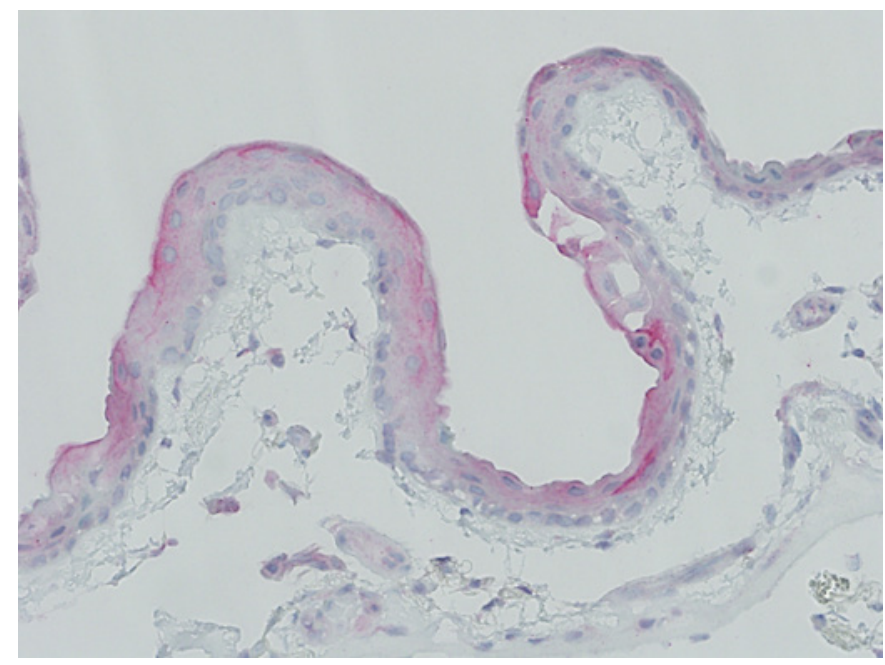

Fig. 3. Immunohistochemical staining of ACE-2 in conjunctival epithelium.

\section{Electron Microscopy}

Postmortem analysis of the macular region of left eye of case 1 (left eye) by electron transmission microscopy yielded noninterpretable results due to the strongly artificially changed material by formalin fixation and paraffin embedding.

\section{Discussion/Conclusion}

As part of a comprehensively analyzed autopsy cohort of 21 COVID-19 patients in the area of Basel [8], 5 patients with ten eyes were examined concerning ocular manifestations. All patients suffered from different comorbidities and died from COVID-19-related respiratory insufficiency. As expected, major morphological findings pertaining to the cause of death were localized in the respiratory tract and were accompanied by a microangiopathic syndrome including pulmonary capillarostasis and presence of fibrin microthrombi in the alveolar capillaries and pulmonary thromboembolism despite anticoagulation [8]. Recent studies reproduce a general prothrombotic state in COVID-19 with in situ clot formation in small vascular beds of different organs [9]. These findings are explained by an impairment of the microcirculatory function by systemic $[1,10,11]$ or local inflammatory endothelial damage [12].

Indeed, in our study, endothelial damage and microthrombi could also be detected in eight of ten eyes. On the other hand, all eyes of the 5 patients showed only patientspecific ocular changes corresponding to the respective pre-existing conditions. There was no evidence of SARS$\mathrm{CoV}$-2-associated inflammatory processes in the neural tissue, the iris, or the corneal tissue. The supremely mild conjunctival inflammatory cell infiltration did not differ from an otherwise usual postmortem finding, especially since these patients all passed away after intensive care, which is known to be linked to an unspecific conjunctival chemosis/injection [13].

On immunohistological analysis, we found fibrin microthrombi in 4 patients mainly in the small choriocapillaris and some in the larger choroidal vessels. Sporadically microthrombi were detected in retinal vessels and ciliary body vessels. No microthrombi were found in the control group, which supports the statement that they are linked to COVID-19.

Fibrin microthrombi may also be caused by sepsis or severe infections like Disseminated Intravascular Coagulation. In the control group, 4 eyes of 2 patients were examined, who died of cholangiosepsis and bronchopneumonia. Fibrin microthrombi could not be found there. The group is small, but this supports our hypothesis that the fibrin microthrombi are specific to SARS-CoV-2.

To our present knowledge, such observations have not been reported so far in eyes. Different studies reported a possible infection of the endothelial cells by SARS-CoV-2 with impaired function and facilitation of local microthrombosis in other organs $[8,12]$, which may be the mechanism beyond our observation in the microcirculation of the eyes.

Fittingly, in most choroid vessels with fibrin microthrombi, some distinct swollen endothelial cells, accumulation of inflammatory cells (mononuclear and neutrophilic) as well as increased apoptotic activity were observable. Finally, in one patient with microthrombosis, low SARS-CoV-2 RNA levels were detectable in the eyes.

It is well known that the SARS-CoV-2 enters the cells by using the ACE- 2 as receptor $[2,3]$. We could show that ACE-2 is over-expressed in the choroidea endothelial cells of choriocapillaris and some of the larger vessels in COVID-19 compared to controls, one may assume that the vascular endothelial system in such instances is generally more susceptible to virus spread.

Indeed, for example, in lungs, there is a 25 -fold increase in the number of ACE-2 positive alveolar epithelial cells in COVID-19 compared to influenza damage [9]. As explained, SARS-CoV-2 can infect microvessels of the human eye via ACE-2 [12].

Additionally, we could show a distinct expression of ACE-2 in the epithelium of the conjunctiva as well as in the epithelium and endothelium of the cornea (shown in Fig. 3). Correspondingly, it has been suggested that SARS- 
CoV-2 can be transmitted through the conjunctiva [14, 15]. Further investigations are needed to find out to which extent the presence of ACE-2 is relevant for virus cell entry into the conjunctiva.

In summary, the analyzed autopsy cohort of eyes from COVID-19 patients demonstrates an impairment of the microvessels mainly in the choroid. There is a local damage of the endothelial cells, which may be infected at low levels by SARS-CoV-2, and overexpress ACE-2 in COVID-19. No high-grade inflammatory process in the neural tissue, the iris, and the corneal tissue and in the conjunctiva is found.

\section{Acknowledgements}

We are thankful to Valeria Perrina, Martin Herzig, Jan Schneeberger, Anna Stalder, and Ralph Schoch (Institute of Medical Genetics and Pathology) for the excellent technical assistance.

\section{Statement of Ethics}

Sample collection was approved by the Ethics Committee of Northwestern and Central Switzerland (ID 2020-00629), which is compatible with the World Medical Association Declaration of Helsinki and goes even further. Consents of the next of kin were obtained that the organs collected at autopsy may be used for research purposes.

\section{Conflict of Interest Statement}

The authors have no conflicts of interest or funding to declare in regard to this study.

\section{Funding Sources}

This work is supported by the Botnar Research Center for Child Health, BRCCH. The sponsor has donated money for the research, without any further influence.

\section{Author Contributions}

Aja Reinhold and Peter Meyer contribute to the conception, analysis and interpretation of data for the work, and writing the manuscript. Peter Meyer, Aja Reinhold, Daniela Mihic-Probst, and Alexandar Tzankov evaluated the ophthalmopathological results and presented them critically regarding important intellectual contents. Alexandar Tzankov and Matthias Matter conducted the PCR and ISH, corrected the manuscript, and drew conclusions about lung involvement in the overall study. Peter Meyer, Alexandar Tzankov, Daniela Mihic-Probst, Aja Reinhold, Matthias Matter, and Hendrik P. N. Scholl finally approved the version to be published and requested agreement to be accountable for all aspects of the work to ensure that issues regarding the accuracy or integrity of any part of the work are properly investigated and resolved.

\section{References}

1 Albini A, Di Guardo G, Noonan DM, Lombardo M. The SARS-CoV-2 receptor, ACE-2, is expressed on many different cell types: implications for ACE-inhibitor- and angiotensin II receptor blocker-based cardiovascular therapies. Intern Emerg Med. 2020;15:75966.

2 Li W, Moore MJ, Vasilieva N, Sui J, Wong SK, Berne MA, et al. Angiotensin-converting enzyme 2 is a functional receptor for the SARS coronavirus. Nature. 2003;426(6965):450-4.

3 Letko M, Marzi A, Munster V. Functional assessment of cell entry and receptor usage for SARS-CoV-2 and other lineage $B$ betacoronaviruses. Nat Microbiol. 2020;5(4):562-9.

4 Hamming I, Timens W, Bulthuis ML, Lely AT, Navis G, van Goor H. Tissue distribution of ACE2 protein, the functional receptor for SARS coronavirus. A first step in understanding SARS pathogenesis. J Pathol. 2004;203(2): 631-7.

5 Savaskan E, Löffler KU, Meier F, MüllerSpahn F, Flammer J, Meyer P. Immunohistochemical localization of angiotensin-converting enzyme, angiotensin II and AT1 receptor in human ocular tissues. Ophthalmic Res. 2004;36(6):312-20.

6 Seah I, Agrawal R. Can the coronavirus disease 2019 (COVID-19) affect the eyes? A review of coronaviruses and ocular implications in humans and animals. Ocul Immunol Inflamm. 2020:1-5.

7 Wu P, Duan F, Luo C, Liu Q, Qu X, Liang L, et al. Characteristics of ocular findings of patients with coronavirus disease 2019 (COVID-19) in Hubei Province, China. JAMA Ophthalmol. 2020;138:575-8.

8 Menter T, Haslbauer JD, Nienhold R, Savic S, Hopfer H, Deigendesch N, et al. Post-mortem examination of COVID19 patients reveals diffuse alveolar damage with severe capillary congestion and variegated findings of lungs and other organs suggesting vascular dysfunction. Histopathology. 2020;77:198-209.

9 Ackermann M, Verleden SE, Kuehnel M, Haverich A, Welte T, Laenger F, et al. Pulmonary vascular endothelialitis, thrombosis, and angiogenesis in covid-19. N Engl J Med. 2020;383:120-8.

10 Tang N, Li D, Wang X, Sun Z. Abnormal coagulation parameters are associated with poor prognosis in patients with novel coronavirus pneumonia. J Thromb Haemost. 2020;18(4): 844-7.

11 Han H, Yang L, Liu R, Liu F, Wu KL, Li J, et al. Prominent changes in blood coagulation of patients with SARS-CoV-2 infection. Clin Chem Lab Med. 2020.

12 Varga Z, Flammer AJ, Steiger P, Haberecker M, Andermatt R, Zinkernagel AS, et al. Endothelial cell infection and endotheliitis in COVID-19. Lancet. 2020;395(10234):1417-8.

13 Loffler KU, Reinhold A, Herwig-Carl MC, Tzankov A, Holz FG, Scholl HPN, et al. [Ocular post-mortem findings in patients having died from COVID-19]. Ophthalmologe. 2020;117:648-51.

14 Napoli PE, Nioi M, d'Aloja E, Fossarello M. The ocular surface and the coronavirus disease 2019: does a dual "ocular route" exist? J Clin Med. 2020;9(5):1269.

15 Chen L, Liu M, Zhang Z, Qiao K, Huang T, Chen $\mathrm{M}$, et al. Ocular manifestations of a hospitalised patient with confirmed 2019 novel coronavirus disease. Br J Ophthalmol. 2020; 104(6):748-51. 